\title{
Heartbeat perception in panic disorder: a reanalysis
}

\author{
A.J. Willem Van der Does ${ }^{\mathrm{a}, *, 1}$, Martin M. Antony ${ }^{\mathrm{b}}$, Anke Ehlers ${ }^{\mathrm{c}}$, \\ Arthur J. Barsky ${ }^{\mathrm{d}}$ \\ ${ }^{a}$ Departments of Psychology and Psychiatry, Leiden University, Wassenaarseweg 52, 2333 AK Leiden, The Netherlands \\ ${ }^{\mathrm{b}}$ Department of Psychology, St. Joseph's Hospital, Hamilton, Ont., Canada \\ ${ }^{\mathrm{c}}$ Department of Psychiatry, Oxford University, Oxford, UK \\ ${ }^{\mathrm{d}}$ Department of Psychiatry, Harvard Medical School, Boston, MA, USA
}

Accepted 1 October 1998

\begin{abstract}
This article describes a reanalysis of seven studies on heart beat perception (HBP) in panic disorder. The pooled sample had 709 participants from eight diagnostic categories. Accurate HBP was uncommon, but more prevalent among panic disorder patients than among healthy controls, depressed patients, patients with palpitations and individuals with infrequent panic attacks. No differences were found between panic disorder patients and patients with other anxiety disorders. Accurate perceivers had higher anxiety sensitivity scores than inaccurate perceivers. The data remain inconclusive as to whether perceived heart rate is correlated with anxiety in inaccurate perceivers. Physical exercise, distraction, variations in instructions and treatment each influenced HBP. However, the influence was different than previously thought. Finally, it is suggested that HBP may be understood in terms of schema-guided information processing. (C) 1999 Elsevier Science Ltd. All rights reserved.
\end{abstract}

\section{Introduction}

There has been some controversy regarding the question of whether patients with panic disorder are better at perceiving their own heartbeats than are other groups. In a heartbeat perception (HBP) task, participants are asked to silently count all heartbeats they feel in their body, without taking their pulse. This procedure is known as 'mental tracking' (Schandry,

\footnotetext{
* Corresponding author. Tel.: +31-71-527-3754; fax: +31-71-527-3619; e-mail: avddoes@pobox.leidenuniv.nl

${ }^{1}$ Address until February 1, 1999: Department of Psychology, Harvard University, William James Hall, 33 Kirkland Street, Cambridge, MA 02138, USA. Tel.: +1-617-495-8033; fax: +1-617-495-3728; e-mail: wvdoes@wjh. harvard.edu
}

0005-7967/99/\$ - see front matter (C) 1999 Elsevier Science Ltd. All rights reserved.

PII: S0005-7967(98)00184-3 
1981). Various other procedures exist to measure HBP, e.g. asking subjects to choose their own heart rhythm from a number of series of tone pips (e.g. Brener \& Kluvitse, 1988). However, because the 'mental tracking' procedure is the only procedure that has yielded significant differences between panic disorder patients and other groups, this article will only concern studies that used this procedure. In the first of these studies, Ehlers and Breuer (1992) showed that panic disorder (PD) patients perform better on this test than normal controls, infrequent panickers and patients with specific phobias. Test performance can be expressed in a continuous score (percentage error) or in a dichotomous score (accurate/nonaccurate, using a criterion of maximum difference between actual and counted beats). The above findings appeared with both types of scores. In a second study, both panic disorder and generalized anxiety disorder patients had lower percentage error scores than depressed patients (Ehlers \& Breuer, 1992). These results were replicated by Ehlers et al. (1995). In this latter study, panic disorder patients again had a significantly lower error score than controls. The group difference was also significant in a second condition, when a series of distracting tones was presented during the task. However, during a third, strict instruction condition ('count only those heartbeats about which you are sure') no group differences emerged. The findings gained clinical relevance in a study by Ehlers (1995), that showed that good HBP was predictive of poor treatment outcome and of recurrence of panic attacks after initial remission.

However, Antony et al. (1995) failed to find differences in HBP among groups of patients with panic disorder, patients with social phobia and normal controls. The error scores of the PD and control group were quite comparable to Ehlers et al. (1995), but with less statistical power the difference was not significant. Furthermore, the social phobics had identical scores to the PD patients.

Barsky, Cleary, Sarnie and Ruskin (1994) studied three groups: two groups of patients who were referred to ECG monitoring for the evaluation of palpitations (groups with and without panic disorder) and a normal comparison group. Results showed that palpitation patients with PD were not more accurately aware of their heartbeats than nonpanic palpitation patients or controls.

Recently, Van der Does, Van Dyck and Spinhoven (1997) found no differences in error scores among patients with panic disorder, depressed patients and normal controls. The above reviewed studies are summarized in Table 1.

Van der Does et al. (1997) argued that comparing mean error scores between groups may not be the best way of analyzing the findings. This has to do with the validity of the HBP task. Although the test-retest reliability of the test is adequate (Ehlers \& Breuer, 1992; Van der Does et al., 1997), its validity is uncertain. Ehlers et al. (1995) found support for the validity in the fact that there is a significant correlation between stroke volume and test results (Schandry, Bestler, \& Montoya, 1993). Furthermore, almost all participants count fewer beats than actually occur, which is a logical outcome if participants accurately count their heartbeats but miss a few. Third, the possibility that participants estimate the duration of the trials and then make an educated guess about the number of heartbeats has been ruled out in several studies (e.g. Ehlers \& Breuer, 1992). However, time estimation is only one of several alternative explanations of HBP test performance. Furthermore, participants' self-reports after the test are contradictory to the second argument. Participants typically report not having missed a few beats, but instead having felt a regular rhythm somewhat slower than the actual rhythm (Van 
Table 1

Summary of the results of mental tracking studies

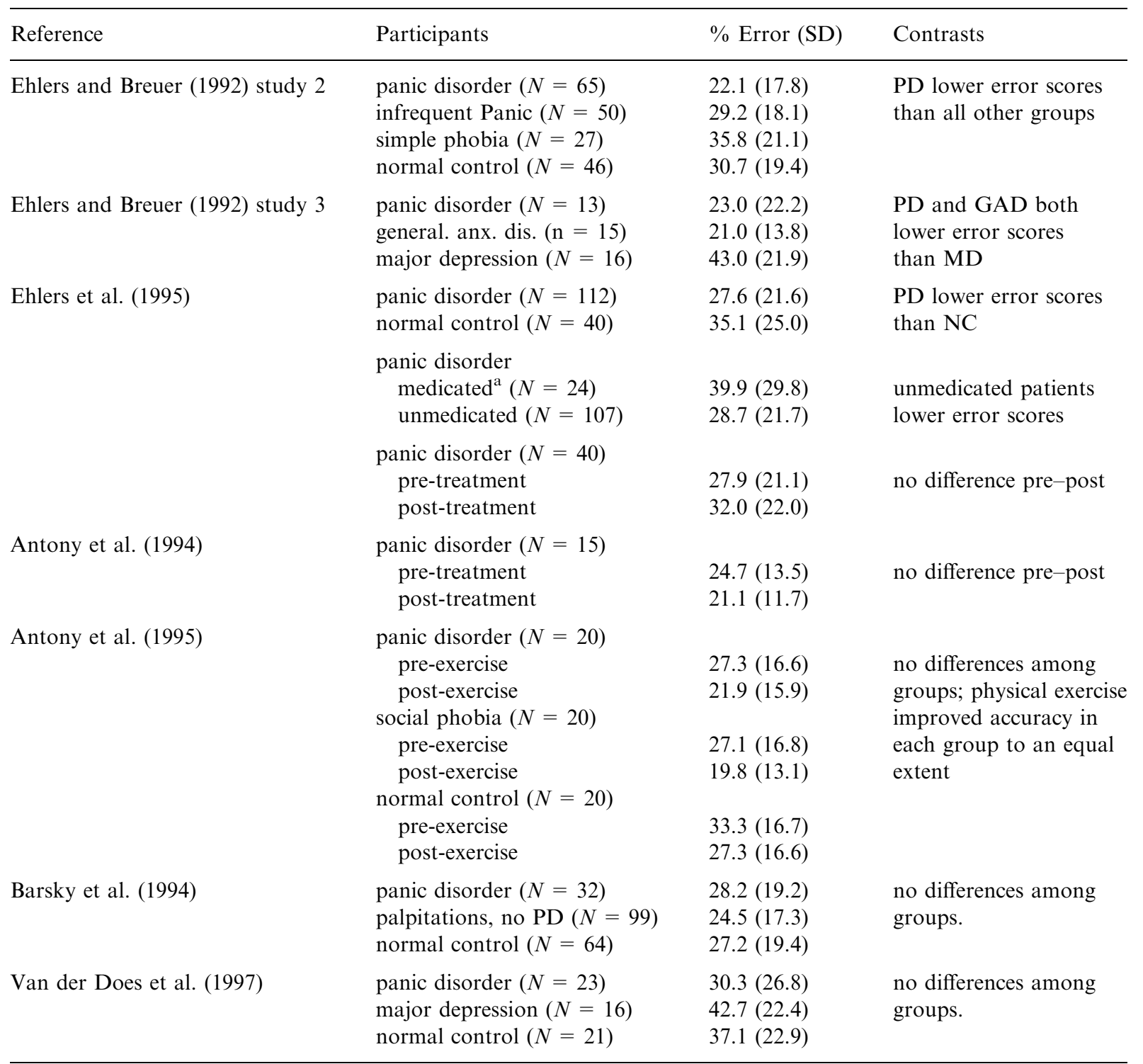

Abbreviations: PD is panic disorder, GAD generalized anxiety disorder, MD major depression, NC normal controls. Tricyclic antidepressants, $\beta$-blockers or other anti-hypertensive medication.

der Does et al., 1997). This makes it uncertain whether the perceived rhythm has anything to do with actual heartbeats. If, hypothetically, PD patients feel more nervous during the test, they may expect and count a faster heartbeat rhythm than normals. They would then get lower error scores, just because almost all participants underestimate their HRs. Alternatively, the lower error scores of PD patients may be caused by a small number of patients who accurately 
perceive their heartbeats (i.e. who have a $0 \%$ error score). Van der Does et al. (1997) therefore looked at individual test performance to decide whether a participant is an accurate perceiver. While the mean error scores were not significantly different among groups, more panic patients $(7 / 23)$ accurately perceived their HRs than depressed patients $(0 / 16)$ or normals $(2 / 21)$. The nine accurate perceivers had higher anxiety sensitivity scores than nonaccurate perceivers. Within the latter group, perceived HR was unrelated to actual HR, unrelated to cognitive scores, but significantly related to state anxiety.

As noted above, the division of participants into accurately and nonaccurately perceiving groups was first used by Ehlers and Breuer (1992), who had also noted that the majority of PD patients were not totally accurate. In fact, the findings by Ehlers and Breuer and by Van der Does et al. (1997) are quite similar. There is a difference in interpretation that is based on the assessment of whether or not the HBP test is a valid measure of heart rhythm. If the test is valid, a division into accurate/nonaccurate creates artificial boundaries. Since Ehlers and Breuer (1992) regard the test as valid, in all subsequent publications (reviewed above) only the continuous measure (\% error) was used. Van der Does et al. (1997) instead, found support for the position that the test is valid for only a small minority of participants, who accurately perceive their heart beats. Most of the remaining participants did come up with a heart beat count, but this was unrelated to actual heart beats. In other words, the division may not be artificial. However, these findings may be dependent on the relatively small sample that was used in that study. Therefore, the authors of the studies listed in Table 1 decided to collaborate in a reanalysis. The data of these studies were pooled and reanalyzed. The first question that was addressed was whether the main findings by Van der Does et al. (differences in anxiety sensitivity scores between accurate/nonaccurate perceiving subjects and correlation with state anxiety) replicate in a larger sample. A number of additional questions was addressed. Firstly, Antony et al. (1995) tested their participants both at rest and after a period of physical exercise, and found that exercise improved the mean accuracy scores in all groups. It would be interesting to know whether this was due to improvement in the ratio accurate/nonaccurate perceivers or to an increase in anxiety or arousal level in nonaccurate perceivers. Secondly, it would be interesting to see whether the confidence ratings, as used in several studies, are different for accurate and nonaccurate perceivers. Thirdly, Ehlers et al. (1995) used two types of instructions as well as a distraction procedure. Distraction had little influence on the percentage error scores, whereas strict instructions did have influence. It is unknown whether this also applies to the dichotomous scores. A final question concerned the effect of treatment on accuracy of HBP. On the basis of percentage error scores before and after treatment, it has been concluded that good HBP is a rather stable individual characteristic (Antony et al., 1994; Ehlers et al., 1995).

\section{Method}

\subsection{Participants}

The pooled data set contained a total of 709 participants, with the following diagnoses: panic disorder $(N=275)$, palpitation patients (without panic disorder) $(N=99)$, infrequent 
panic $(N=50)$, mood disorder $(N=32)$, specific phobia $(N=27)$, social phobia $(N=20)$, generalized anxiety disorder $(N=15)$, normal controls $(N=191)$.

\subsection{Procedure}

In each study, HBP was assessed with the mental-tracking procedure (Schandry, 1981). Participants were instructed to silently count their heartbeats during signalled intervals without taking their pulse. Participants had to take their watches off and the length of the intervals was unknown to them. In the study of Barsky et al. (1994) two trials were administered (15 s each), and participants estimated their HRs at home, while wearing an ambulatory ECG monitor. In the other studies, participants were tested in the laboratory. In Antony et al. $(1994,1995)$ seven trials per condition were used, lasting between 14 and 22 s. Three trials per condition were used in the studies by Ehlers et al. and by Van der Does et al. (35, 25 and $45 \mathrm{~s})$.

\subsection{Questionnaires}

Each separate study had its own set of questionnaires, and no questionnaire was used in all studies. The following questionnaires were used frequently enough to justify inclusion in this reanalysis. Anxiety disposition and state anxiety were measured with the State-Trait Anxiety Inventory (STAI) (Spielberger, Gorusch, \& Lushen, 1970). The Body Sensations Questionnaire (BSQ) and Agoraphobic Cognitions Questionnaire (ACQ) (Chambless et al., 1984) were used to measure the fear of body sensations and the tendency to have catastrophic thoughts while being nervous or anxious. The Anxiety Sensitivity Index (ASI) (Peterson \& Reiss, 1992) was used to measure the beliefs about the harmfulness of bodily sensations. The tendency to amplify somatic sensations was measured using the Somatosensory Amplification Scale (SSAS) (Barsky et al., 1990). In several studies, participants rated their confidence in their heartbeat estimation accuracy on a $0-10$ or $0-100$ scale. These scores were all transformed to a $0-100$ scale. In Antony et al. (1994, 1995), participants also rated physical arousal after each trial and pre- and posttrial anxiety, each on a 0-100 scale.

\subsection{Data processing and analysis}

Prior to analysis, the data were examined for accuracy of data entry, missing values and normality of distributions, following the recommendations by Tabachnick and Fidell (1989). Between-group differences were examined with oneway analysis of variance and Bonferronicorrected LSD contrasts (or $\chi^{2}$ tests when appropriate). Partial correlation coefficients were calculated to investigate the relationships between perceived HRs and questionnaire scores. Actual HRs were partialled out, because these had a significant correlation with perceived HR in each group (around 0.30). 


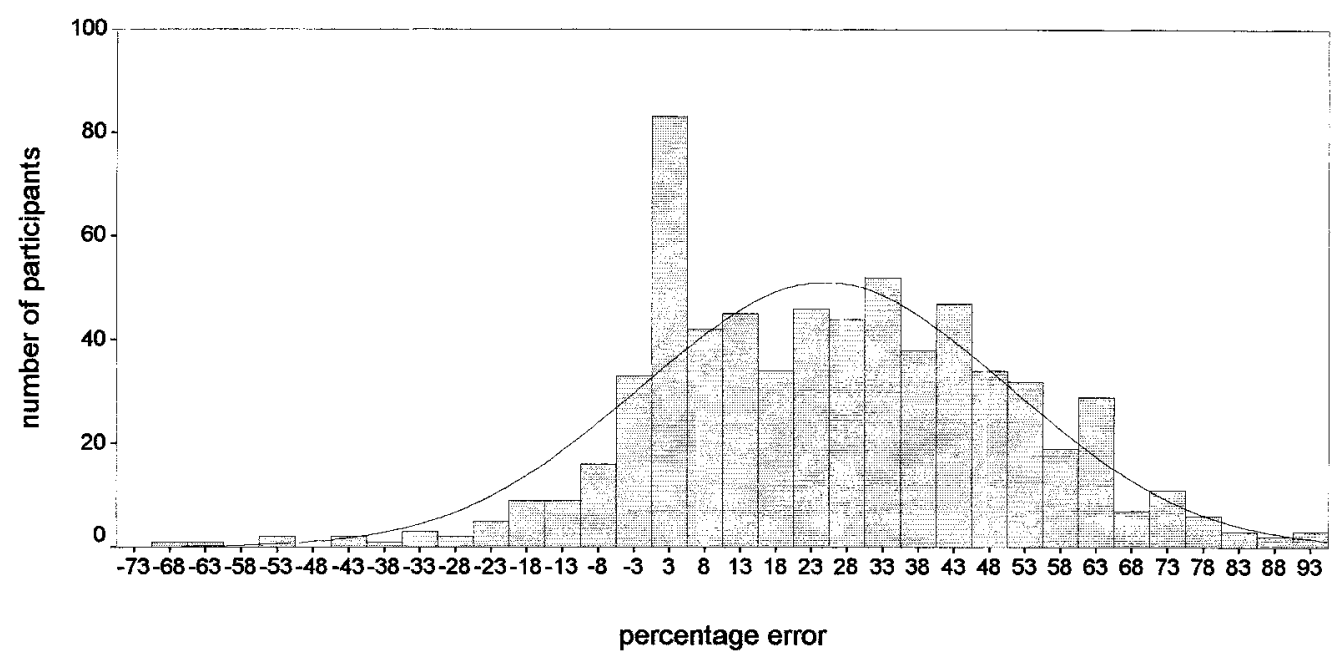

Fig. 1. Histogram of percentage error scores, preintervention trial (normal curve superimposed). $N=663$.

\section{Results}

\subsection{Distribution of error scores}

Fig. 1 shows the distribution of error scores on the first trial for all participants combined. The scores are normally distributed, with a marked and skewed peak around $0 \%$ error. Fig. 2-3 show the histograms of the 'distraction' and 'strict instruction' conditions, which both appear bimodal.

\subsection{Definition of accurate perception}

Participants were then divided into accurate or nonaccurate perception groups on the basis of their individual error scores at each trial. This posed a problem, because the number of trials (preintervention) varied among studies, from two (Barsky et al. (1994)) to three (Ehlers, Van der Does) to seven (Antony et al. (1994, 1995)). What to do, for instance, with a participant who had 3\% error in four out of seven trials or a participant from the studies of Barsky who had $0 \%$ error in the first trial but felt nothing (or with $11 \%$ error, or missing data) in the second trial? Three categories were created (accurate, probably accurate, inaccurate), using the following rules.

A participant was considered an accurate perceiver if he or she had less than $10 \%$ error in each of two or three trials or in at least five of seven trials.

A participant was considered probably accurate if he or she had: (a) less than $10 \%$ error in one of two trials, but only if the second trial was less than $20 \%$ error or was missing, (b) less than $10 \%$ error in two of three trials, (c) less than $10 \%$ error in one of three trials, but only if the other two trials were less than $20 \%$ wrong or were missing and (d) less than $10 \%$ error in four of seven trials. 


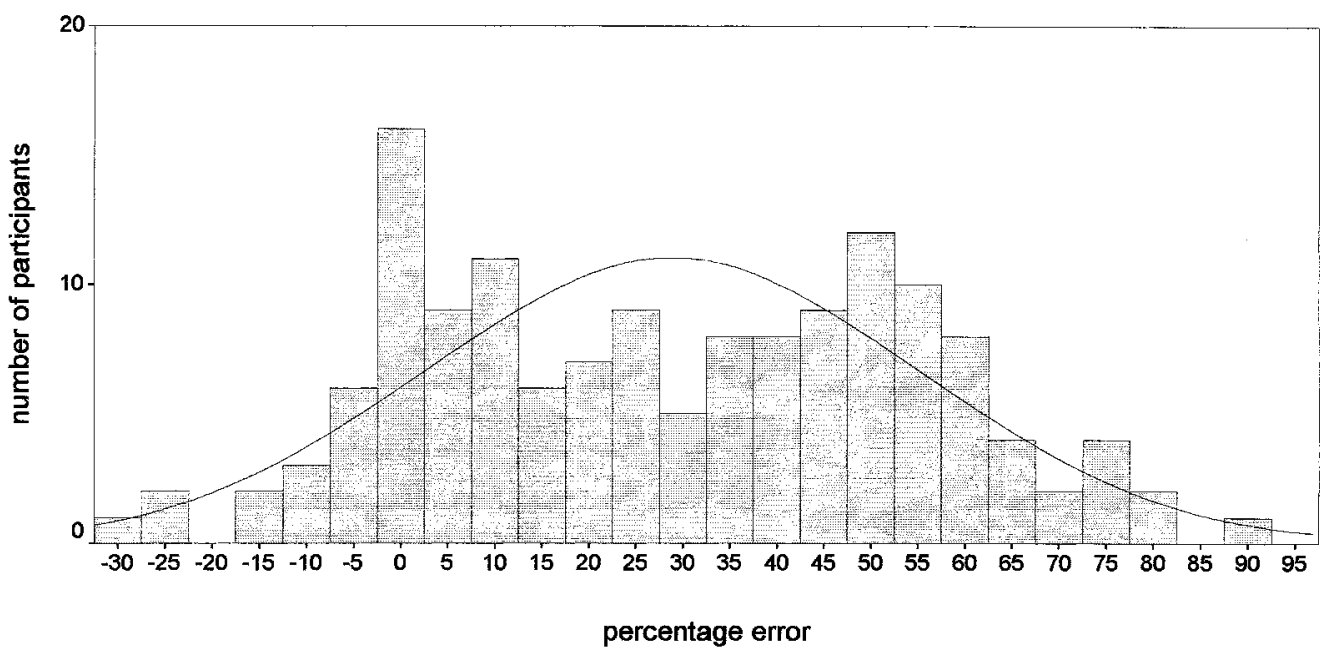

Fig. 2. Histogram of percentage error scores, distraction trial (normal curve superimposed). $N=145$.

All remaining participants were considered inaccurate perceivers. The $10 \%$ error criterion was chosen because it allows an error margin of two beats during shorter trials (this is the criterion used by Schandry (1981)) and a slightly higher margin during longer trials.

\subsection{Distribution of accurate perceivers}

The distribution of accurate and nonaccurate perceivers is shown in Table 2. $\chi^{2}$ analysis showed that the distribution is significantly different among groups. Subsequent one-by-one analyses showed that there are more accurate perceivers in the PD group than among the normal controls, the depressed patients and among the patients with palpitations. These

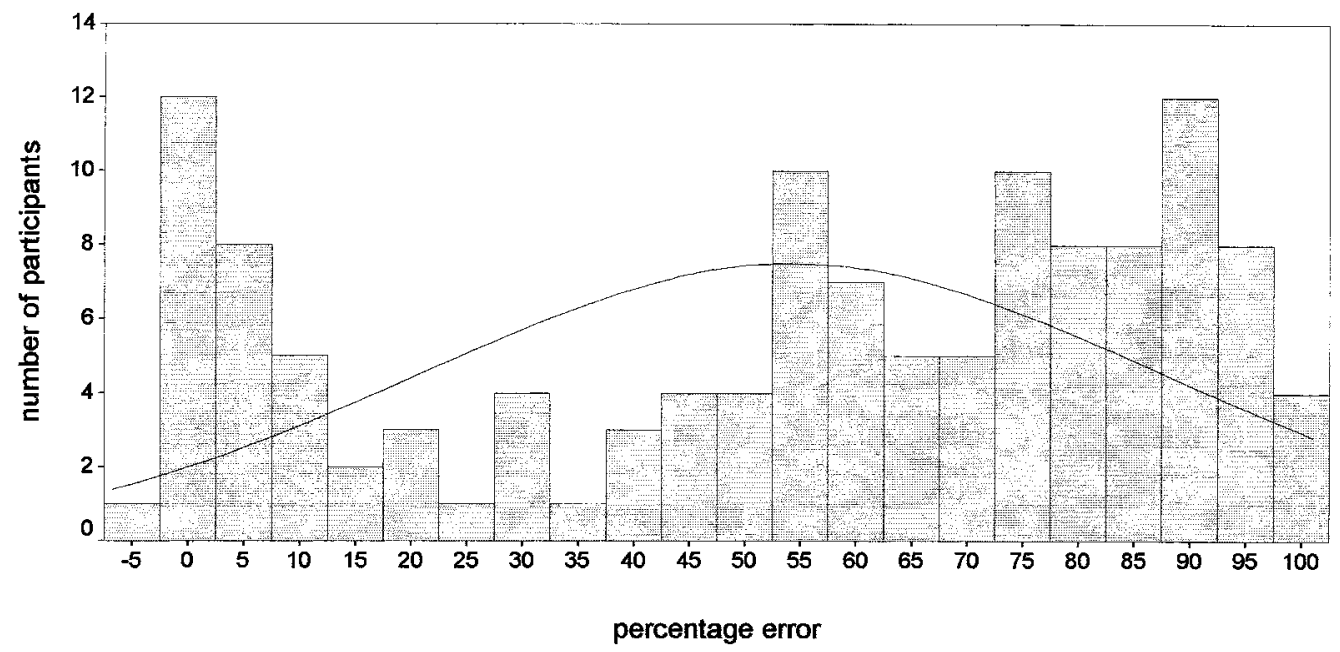

Fig. 3. Histogram of percentage error scores, strict instruction trial (normal curve superimposed). $N=125$. 
Table 2

Reanalysis of pooled data

\begin{tabular}{|c|c|c|c|c|}
\hline & \multicolumn{3}{|c|}{ Accurate perception $(\%$ of $\mathrm{Ss})$} & \multirow[t]{2}{*}{ Comparisons } \\
\hline & no & probable & yes & \\
\hline \multicolumn{5}{|l|}{ Preintervention } \\
\hline Panic disorder $(N=275)$ & 68.4 & 14.5 & 17.1 & all groups: $\chi^{2}(14)=29.4 ; \mathrm{p}=0.009$ \\
\hline Normal control $(N=191)$ & 79.1 & 13.1 & 7.9 & $\mathrm{PD}$ vs. $\mathrm{NC}: \chi^{2}(2)=9.2 ; \mathrm{p}=0.01$ \\
\hline Palpitations, no PD $(N=99)$ & 74.7 & 19.2 & 6.1 & PD vs. Palps: $\chi^{2}(2)=7.7 ; \mathrm{p}=0.02$ \\
\hline Infrequent panic $(N=50)$ & 82.0 & 12.0 & 6.0 & PD vs. Infr.: $\chi^{2}(2)=4.7 ; p=0.10^{a}$ \\
\hline Mood disorder $(N=32)$ & 90.6 & 9.4 & 0.0 & PD vs. $\mathrm{MD}: \chi^{2}(2)=8.3 ; \mathrm{p}=0.02$ \\
\hline Specific phobia $(N=27)$ & 77.8 & 11.1 & 11.1 & PD vs. $\mathrm{SpPh}: \chi^{2}(2)=1.0 ; \mathrm{p}=0.59$ \\
\hline Social phobia $(N=20)$ & 70.0 & 5.0 & 25.0 & PD vs. SocPh: $\chi^{2}(2)=1.9 ; \mathrm{p}=0.39$ \\
\hline General. anx. disorder $(N=15)$ & 73.3 & 6.7 & 20.0 & PD vs. GAD: $\chi^{2}(2)=0.7 ; \mathrm{p}=0.69$ \\
\hline \multicolumn{5}{|l|}{ Postexercise } \\
\hline Panic disorder $(N=20)$ & 30.0 & 45.0 & 25.0 & All groups: $\chi^{2}(4)=2.4 ; p=0.67$ \\
\hline Normal control $(N=20)$ & 50.0 & 40.0 & 10.0 & \\
\hline Social phobia $(N=20)$ & 40.0 & 40.0 & 20.0 & \\
\hline \multicolumn{5}{|l|}{ Posttreatment } \\
\hline Panic disorder $(N=79)$ & 63.3 & 19.0 & 17.7 & \\
\hline \multicolumn{5}{|l|}{ Distraction trials } \\
\hline Panic disorder $(N=112)$ & 72.3 & 14.3 & 13.4 & $\chi^{2}(2)=0.9 ; \mathrm{p}=0.63$ \\
\hline Normal control $(N=40)$ & 78.9 & 13.2 & 7.9 & \\
\hline \multicolumn{5}{|l|}{ Strict instruction trials } \\
\hline Panic disorder $(N=112)$ & 81.7 & 11.5 & 6.7 & $\chi^{2}(2)=2.2 ; \mathrm{p}=0.33$ \\
\hline Normal control $(N=40)$ & 87.9 & 3.0 & 9.1 & \\
\hline
\end{tabular}

${ }^{\text {a }}$ With accuracy recoded as dichotomous variable (inaccurate and probably accurate taken together), this comparison was significant: $\chi^{2}(1)=4.0, p=0.05$. This applied to none of the other comparisons.

analyses were repeated using dichotomous scores ('probable' group merged with inaccurate group). This yielded another significant difference, between the PD and Infrequent panic groups $\left(\chi^{2}(1)=4.0, p<0.05\right)$. All other results were unchanged.

\subsection{Distribution of 'nonresponders'}

Only 25 participants of the total sample (3.5\%) reported feeling no heartbeat at all during the preintervention trials. This applied to only three $(1.1 \%)$ of the panic disorder patients and to none of the patients with social phobia, infrequent panic, specific phobia or GAD. Six $(6.1 \%)$ of the patients with palpitations, six (18.8\%) of the depressed patients and $10(5.2 \%)$ of the normal controls felt nothing during the trials. The distribution was significantly different among groups $\left(\chi^{2}(7)=34.2, p<0.0001\right)$. One-by-one analyses showed that there were fewer 
Table 3

Accurate versus nonaccurate perceivers, all diagnostic groups combined

\begin{tabular}{lcccc}
\hline \multicolumn{4}{c}{ Accurate perception } \\
\cline { 2 - 4 } & no & probable & yes & \\
\hline Anxiety sensitivity & $21.0^{\mathrm{a}}$ & 21.6 & $26.9^{\mathrm{b}}$ & $F(316,2)=3.8, p=0.02$ \\
& $(12.5)$ & $(14.7)$ & $(15.1)$ & \\
Agoraphobic cognitions & 24.3 & 25.0 & 27.7 & $F(297,2)=2.8, p=0.06$ \\
& $(8.3)$ & $(8.9)$ & $(10.1)$ & \\
\hline
\end{tabular}

Note. Means with different suffixes $(\mathrm{a}, \mathrm{b})$ differ significantly at $p<0.05$ (Bonferroni-corrected LSD contrasts).

'nonresponders' among the panic patients than among the patients with palpitations, the depressed patients and the controls.

\subsection{Characteristics of participants with accurate perception}

Accurate, probably accurate and inaccurate perceivers were compared on a number of demographic variables and questionnaire scores. There were no significant differences in age, body mass index or actual number of heartbeats during the trials. However, $72.8 \%$ of males and $80.6 \%$ of females were inaccurate perceivers, which is a significant difference $\left(\chi^{2}(2)=6.2\right.$, $p=0.04)$. Furthermore, accurate perceivers scored about half a standard deviation higher than inaccurate perceivers on the ASI, but not on any of the other rating scales (e.g. trait and state anxiety, depressive symptoms, amplification). This result is shown in Table 3, in which the data from Van der Does et al. (1997) were left out (the study in which this relationship was first found).

\subsection{Correlates of perceived heart rate}

Van der Does et al. (1997) had found a significant correlation (around 0.50) between the number of perceived beats and anxiety (self-reported state anxiety and skin conductance level), for nonaccurately perceiving participants. This correlation was significant in their whole group of participants as well as in panic disorder patients only (Van der Does et al., 1998). In this reanalysis, Pearson correlations were calculated between the number of counted beats, actual beats, age, body mass index and questionnaire scores. The data from Van der Does et al. (1997) were filtered out, because this part of the reanalysis was concerned with replicating their findings. Only state anxiety and actual HR correlated significantly with the number of counted beats. The partial correlation coefficient (actual HR partialled out) was significant for state anxiety, but not for any of the cognitive measures of panic. However, in contrast with Van der Does et al. (1997), the correlation for state anxiety was negligible. Furthermore, when partial correlations were calculated within diagnostic subgroups, it appeared that the correlation was significant within the infrequent panic group only (see Table 4, top half). It should be noted that these correlations only concern the studies by Ehlers and colleagues. The Van der Does 
Table 4

Correlates of number of perceived heartbeats, preintervention trials (inaccurate perceivers)

\begin{tabular}{|c|c|c|c|c|c|c|c|}
\hline Ehlers et al. studies & $\begin{array}{l}\text { All Ps } \\
(n=215)\end{array}$ & $\begin{array}{l}\mathrm{NC} \\
(n=62)\end{array}$ & $\begin{array}{l}\mathrm{PD} \\
(n=99)\end{array}$ & $\begin{array}{l}\text { Infr.P. } \\
(n=38)\end{array}$ & $\begin{array}{l}\text { Spec.Ph. } \\
(n=16)\end{array}$ & & \\
\hline Age & 0.00 & -0.16 & 0.06 & 0.09 & -0.32 & & \\
\hline Anxiety sensitivity & 0.08 & -0.07 & -0.14 & 0.19 & -0.11 & & \\
\hline State anxiety & $0.11^{*}$ & -0.09 & 0.03 & $0.40^{* *}$ & -0.18 & & \\
\hline \multirow[t]{2}{*}{ Antony et al. studies } & \multicolumn{7}{|c|}{ panic disorder patients $(n=22)$} \\
\hline & triall & trial2 & trial3 & trial4 & trial5 & trial6 & trial7 \\
\hline Physical arousal (self-report) & -0.02 & 0.09 & 0.05 & -0.01 & 0.07 & 0.03 & 0.04 \\
\hline Pretrial anxiety (self-report) & $0.46^{*}$ & $0.58^{* *}$ & $0.62^{* * *}$ & $0.54^{* *}$ & $0.42^{*}$ & $0.53^{* *}$ & $0.42^{*}$ \\
\hline Posttrial anxiety (self-report) & $0.60^{* *}$ & $0.64^{* * *}$ & $0.65^{* * *}$ & $0.60^{* *}$ & $0.51^{* *}$ & $0.58^{* *}$ & $0.51^{* *}$ \\
\hline
\end{tabular}

Notes: Partial correlations (actual HR partialled out). One-tailed significance: ${ }^{*} p<0.05 ; \quad{ }^{* *} p<0.01$; ${ }^{* * *} p<0.001$.Abbreviations: Ps is participants, NC normal controls, PD panic disorder, Infr.P. infrequent panic, Spec.Ph. specific phobia.

data were filtered out because of the above mentioned reason; the data of Barsky et al. (1994) because the STAI was not administered on the same day as the HBP test; the data of Antony et al. $(1994,1995)$ because anxiety was not measured with the STAI.

The relationship between perceived beats and anxiety could be further investigated because in the studies of Antony et al. (1994, 1995) participants had given subjective arousal and anxiety ratings at each separate trial. Both pre- and posttrial anxiety, but not physical arousal, were significantly related with counted beats (around 0.50), except for the first two trials. Partial correlations were then calculated within each diagnostic subgroup. This yielded no significant results for the normal controls and social phobics, whereas for the PD group, correlations were significant at each trial (see Table 4, bottom half).

\subsection{Effects of physical exercise}

Antony et al. (1995) had shown that physical exercise improves percentage error scores over baseline trials for the first four of seven trials following exercise. This effect was the same for patients with panic disorder, social phobics (SP) and normal controls (NC). Reanalysis using accurate/nonaccurate score patterns showed that exercise had a short-term effect on HBP for 25 of the 60 participants. These participants had very low error scores during the first two or three trials following exercise, followed by a sharp increase at the third or fourth trial. These participants were placed in a separate category, which was labelled 'partially accurate' (rather than 'probably accurate'). Further analyses with these category definitions showed that about half $(31 / 60)$ of the participants were in the same category before and after exercise. An enduring increase in accuracy after exercise was quite rare: only one baseline inaccurate perceiver (a PD patient) and only two participants from the 'probable' category before exercise (one NC and one SP) were accurate postexercise. Twenty-two baseline inaccurate perceivers (eight PD, seven SP, seven NC) moved to the partially accurate category postexercise (see 
Table 5

Number of participants with accurate perception before and after physical exercise

\begin{tabular}{lcccr}
\hline Baseline & \multicolumn{2}{l}{ Postexercise } & & \\
\cline { 2 - 5 } & no & partial & yes & total \\
\hline No & 21 & 22 & 1 & 44 \\
Probable & 2 & 2 & 2 & 6 \\
Yes & 1 & 1 & 8 & 10 \\
Total & 24 & 25 & 11 & 60 \\
\hline
\end{tabular}

Table 5). Thus, increased heart rate (HR) through physical exercise had a very short-term effect on about half of the inaccurate perceivers, equally divided over diagnostic groups. The effect is depicted in Fig. 4. A $3 \times 7$ (group by trial) repeated measures analysis of variance with actual HR as covariate showed that the graphs are different among groups: both main effects and the interaction effect $(F[12,335]=6.12, p<0.001)$ were significant. The groups did not differ in actual HR at any trial. In terms of questionnaire scores, the partially accurate group was more like the inaccurate than the accurate group.

The postexercise percentage error scores of inaccurate perceivers were not improved compared to baseline scores, which further illustrates that the effect is due to a selective and transient improvement in the number of accurate perceivers, and not to a general improvement of percentage error scores across participants (as had been concluded by Antony et al., 1995).

The course of the confidence ratings over trials indicates that participants seem to know whether they are really counting their heart beats. At the first trial (real HR around $130 \mathrm{bpm}$ ), the confidence ratings of accurate and partially accurate perceivers were higher than those of inaccurate perceivers. However, from the third trial on (real HR around $95 \mathrm{bpm}$ ), accurate

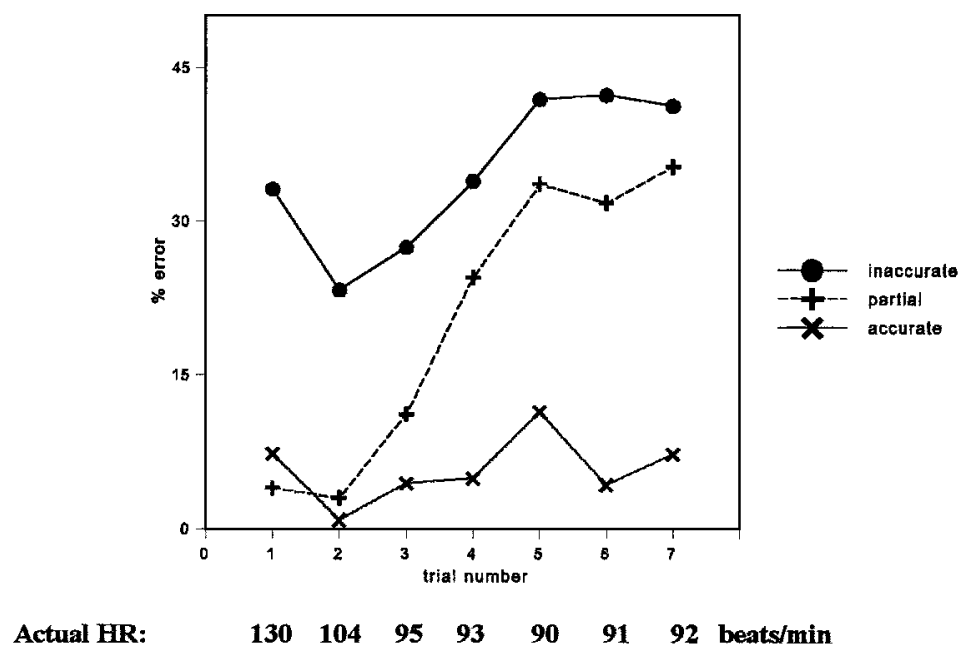

Fig. 4. Percentage error scores and actual heart rates per trial for accurate, partially accurate and inaccurate perceivers, postexercise trials. 
Table 6

Number of panic disorder patients with accurate perception before and after treatment

\begin{tabular}{lcccc}
\hline Baseline & \multicolumn{2}{l}{ Posttreatment } & & \\
\cline { 2 - 5 } & no & probable & yes & total \\
\hline No & 40 & 6 & 5 & 51 \\
Probable & 6 & 4 & 1 & 11 \\
Yes & 4 & 5 & 8 & 17 \\
Total & 50 & 15 & 14 & 79 \\
\hline
\end{tabular}

perceivers were significantly more confident than both other groups. Finally, correlations of perceived HR with subjective ratings of arousal and anxiety were calculated. Interestingly, the pattern and strength of the correlations were very similar to the baseline phase.

\subsection{Effects of treatment}

Pre- and posttreatment data were available for 79 panic disorder patients. There were 17 accurate perceivers before treatment. Less than half of these (8/17) were also accurate after treatment; four patients were inaccurate posttreatment, while five moved to the probable category (see Table 6). In contrast, only five of 51 baseline inaccurate perceivers became accurate perceivers after treatment. The test score differences between accurate and nonaccurate perceivers posttreatment were in the same direction as the pretreatment differences between these groups (higher cognitive panic scores for accurate perceivers). However, none of these differences approached statistical significance.

\subsection{Effects of instructions and distraction}

Forty normal controls and 112 PD patients were tested under standard as well as under distraction conditions. The distraction procedure was a series of tone pips during the HBP test. These 152 participants were also tested under a strict instruction - condition ('count only those heartbeats of which you are sure'). Ehlers et al. (1995) had found that distraction had a minimal effect on the percentage error scores of normals as well as patients. A strict instruction, however, had a substantial deteriorating effect on the performance of both groups. Also, the performance of both groups was no longer different under the strict instruction.

In contrast, reanalysis with categorical scores revealed a larger effect of distraction on performance: of 20 baseline accurate perceivers, seven participants $(35 \%)$ were inaccurate while distracted, and another four (20\%) moved to the probable category. The effect of strict instructions was not very different from the effect of distraction. Table 7 shows both effects. Under all three conditions (standard, distraction, strict), accurately perceiving participants were more confident than inaccurate perceivers. The probably accurate group had confidence ratings close to the accurate group under standard and distraction conditions and close to the inaccurate group under the strict instructions-condition. Even with strict instructions, however, 
Table 7

Number of participants with accurate perception under standard instructions (baseline), distraction and strict instruction

\begin{tabular}{lrrrrrrrr}
\hline Baseline & \multicolumn{2}{l}{ Distraction } & \multicolumn{7}{c}{ Strict instruction } \\
\cline { 2 - 8 } & no & probable & yes & total & no & probable & yes & total \\
\hline No & 93 & 12 & 6 & 111 & 103 & 6 & 2 & 111 \\
Probable & 13 & 5 & 3 & 21 & 17 & 2 & 2 & 21 \\
Yes & 7 & 4 & 9 & 20 & 9 & 5 & 6 & 20 \\
Total & 113 & 21 & 18 & 152 & 129 & 13 & 10 & 152 \\
\hline
\end{tabular}

the overall mean confidence rating (all groups) was only $60.4 \%(\mathrm{SD}=22.9)$, which shows that participants took the instructions rather liberally or found them confusing (for instance, participants who counted a low number of beats may have given low confidence ratings because they knew that their answer could not be correct).

\section{Discussion}

This reanalysis settles some of the discrepancies that arose from the individual studies listed in Table 1. Accurate HBP is more often found among PD patients than among normal controls, depressed patients, patients with palpitations and individuals with infrequent panic attacks. However, no differences were observed between PD patients and patients with social phobia, generalized anxiety disorder or specific phobia. These latter three groups were rather small, however. A higher number of cases could have made a difference for the specific phobia group. The reanalysis confirmed that accurate perception is restricted to a minority of participants in each group (Ehlers \& Breuer, 1992; Van der Does et al., 1997). Furthermore, as in Van der Does et al. (1997), participants with accurate perception had higher anxiety sensitivity scores, which may be the reason why good HBP is predictive of worse outcome (Ehlers, 1995). Thus, accurate HBP is not specific to panic disorder, is uncommon, and is predominantly found among patients who have continuous or frequent episodes of clinical anxiety.

The data remain inconclusive as to whether anxiety and number of perceived heart beats are correlated (in inaccurate perceivers). Reanalysis of the studies of Antony et al. (1994, 1995) (where the most precise measures of pre- and posttrial anxiety were gathered) demonstrated significant correlations between number of counted beats and pre- and posttrial anxiety ratings, in particular for the panic disorder group. These correlations were of the same magnitude as previously reported by Van der Does et al. (1997): around 0.55. However, in the reanalysis of the Ehlers studies, the correlation with the STAI was statistically significant but negligible $(r=0.11)$. Within the panic disorder group, the correlation was not even significant. What to make of this discrepancy? A small procedural difference may be responsible. In the studies by Ehlers and colleagues, the STAI was administered before the HBP test was introduced. In the Antony and Van der Does studies, anxiety ratings were obtained immediately after (or before 
and after) the trials. Therefore, the ratings obtained by Ehlers were more independent of the test than in the other studies. Another explanation may be differences in sample characteristics. State anxiety did correlate with perceived HR in the sample of Ehlers and Breuer (1992) of infrequent panickers (see Table 4). Perhaps the correlation is restricted to a subgroup of PD patients whose main or only worry is heart problems. These explanations are speculative, however. More research is needed to resolve this issue, preferably using both self-report and psychophysiological indices of anxiety.

The effect of increased HR through physical exercise was a temporary increase in the number of accurate perceivers. Again, the effect was not specific to panic disorder. More participants were accurate when actual HR was higher than 100 beats/min. At rates of 95 beats/min., the number of accurate perceivers returned to baseline levels. How representative are these HRs for naturally occurring panic attacks? McNally (1994, p. 21-23) has summarized the available data. It appears that HR increases during panic attacks are rather modest (if they occur at all), and are restricted to situational attacks (e.g. Margraf et al., 1987). This suggests that most patients stay in the HR range during which no increase in accuracy occurs. However, in three other studies (cited in McNally, 1994, p. 24), unprovoked panic attacks recorded in the laboratory were accompanied by abrupt increases in HR for some of the participants. In summary, the HR increases that allowed some participants to become accurate perceivers in the physical exercise study do seem to occur in naturally occurring panic attacks, but certainly not in all.

The fact that the distraction procedure (presenting a series of tones during the test) had a substantial effect on the number of accurately perceiving participants is a very important finding. This may explain why studies that used a different method for measuring HBP namely, having participants match the rate of a series of tone pips to their HR - typically find poor (chance level) performance and no differences among groups (e.g. Ehlers et al., 1988b; Butler \& Rapee, 1991).

On the basis of previous studies comparing pre- and posttreatment scores, it has been concluded that good HBP is a relatively stable interindividual difference (Ehlers \& Breuer, 1996). However, the results of this reanalysis show that this conclusion may have to be reconsidered. Less than half of the accurate PD patients at pretreatment were also accurate after (successful) treatment of their disorder. Inaccurate perception, however, was more stable: less than $10 \%$ of baseline inaccurate perceivers were accurate after treatment.

When more than $95 \%$ of all participants report that they perceive their heart rate, and when $80 \%$ of them appear to be wrong (by approximately $30 \%$ ), what in fact do these individuals feel? As argued by Van der Does et al. (1997), self-reports after the trials indicate that many people feel a regular rhythm, which appears to be somewhat slower than their actual HR. This finding makes it unlikely that participants feel their actual HR but miss a few. It is possible that vague bodily sensations are misinterpreted as heart beats. This would be consistent with the fact that confidence ratings are rather low, also under strict instructions ('count only those heartbeats about which you are sure'). However, the tendency to amplify somatic sensations was measured with the SSAS, and this measure failed to correlate with the number of counted heart beats.

Do these findings fit into the cognitive model of panic? More accurate heart rate perception by patients with panic disorder has been interpreted as in line with the model, because the 
frequent focusing of attention on interoceptive sensations increases the probability for these sensations to be detected (Clark, 1996). However, accurate heart rate perception is not a necessary assumption of the cognitive model (Ehlers et al., 1988a), so the results of this study have no consequences for the validity of the model. The findings can perhaps be understood within a conceptual framework employed in psychosomatic research. According to Pennebaker (1982), symptoms are organized in cognitive schemata. These schemata are formed on the basis of previous experience, and guide the perception and processing of current symptoms. It is conceivable that once a patient with panic disorder perceives a situation as threatening, an 'anxiety' schema is activated, and that perception of symptoms is more guided by the schema (that is, by past information) than based upon present physiological status. This hypothetical anxiety schema would include shifting of attentional focus, selective perception, high HR, other arousal symptoms and anxiety. Voluntary shifting of attentional focus during the HBP task may activate (other components of) the anxiety-schema in PD patients.

If the above hypotheses about schema-guided processing are correct, research could shift to investigating the conditions under which schema-guided versus physiology-based processing occur. A possibly counter-intuitive (and speculative) treatment implication could be that interoceptive training may be helpful to some PD patients. This implication is counter-intuitive because good HBP has been associated with poor treatment outcome (Ehlers, 1995) and because accurately perceiving participants have higher anxiety sensitivity scores. However, only a minority of panic attacks are accompanied by markedly elevated HRs. Patients whose panic attacks are not accompanied by increases in HR (to be determined by ambulatory HR monitoring) but who subjectively report HR increases, may be the ones for whom schemaguided processing occurs and for whom this is problematic. These patients may be helped by interoceptive training or by being informed that what they feel is not their heart.

\section{Conclusions}

(1) Accurate HBP is more prevalent among panic disorder patients than among healthy controls, depressed patients, patients with palpitations and individuals with infrequent panic attacks. No differences were found between panic disorder patients and patients with other anxiety disorders. (2) Accurate HBP is uncommon, even in patients with anxiety disorders. (3) Only very high HRs ( $>100 \mathrm{bpm}$ ) lead to an increase in accuracy, but even then for only half of the subjects. (4) Heart beat perception is influenced by distraction (tone pips), which is problematic for studies that measured HBP by presenting different rhythms. (5) Accurate perception is not stable before and after treatment; inaccurate perception is more stable. (6) It remains unclear whether perceived heart rate is correlated with anxiety in inaccurately perceiving participants.

\section{References}

Antony, M. M., Meadows, E. A., Brown, T. A., \& Barlow, D. H. (1994). Cardiac awareness before and after cognitive-behavioral treatment for panic disorder. Journal of Anxiety Disorders, 8, 341-350. 
Antony, M. M., Brown, T. A., Craske, M. G., Barlow, D. H., Mitchell, W. B., \& Meadows, E. A. (1995). Accuracy of heartbeat perception in panic disorder, social phobia and nonanxious subjects. Journal of Anxiety Disorders, 9, 355-371.

Barsky, A. J., Cleary, P. D., Sarnie, M. K., \& Ruskin, J. N. (1994). Panic disorder, palpitations and the awareness of cardiac activity. Journal of Nervous and Mental Disease, 182, 63-71.

Barsky, A. J., Wyshak, G., \& Klerman, G. L. (1990). The somatosensory amplification scale and its relationship to hypochondriasis. Journal of Psychiatric Research, 24, 323-334.

Brener, J., \& Kluvitse, C. (1988). Heart beat detection: judgements of the simultaneity of external stimuli and heart beats. Psychophysiology, 25, 554-561.

Butler, K. S., \& Rapee, R. M. (1991). The influence of anxiety on accuracy of heart rate estimations. Behavior Change, 3, 117-123.

Chambless, D. L., Caputo, G. C., Bright, P., \& Gallagher, R. (1984). Assessment of fear in agoraphobics: the Body Sensations Questionnaire and Agoraphobic Cognitions Questionnaire. Journal of Consulting and Clinical Psychology, 52, $1090-1097$.

Clark, D. M. (1996). Key-note address. Paper presented at the XXVI Congress of the European Association of Behavioural and Cognitive Therapies. Budapest, Hungary, October.

Ehlers, A. (1995). Factors associated with the maintenance of panic attacks: a one-year prospective study. Journal of Abnormal Psychology, 104, 164-172.

Ehlers, A., \& Breuer, P. (1992). Increased cardiac awareness in panic disorder. Journal of Abnormal Psychology, 101, 371-382.

Ehlers, A., \& Breuer, P. (1996). How good are patients with panic disorder at perceiving their heartbeats? Biological Psychology, 42, $165-182$.

Ehlers, A., Breuer, P., Dohn, D., \& Fiegenbaum, W. (1995). Heartbeat perception and panic disorder: possible explanations for discrepant findings. Behaviour Research and Therapy, 33, 69-76.

Ehlers, A., Margraf, J., \& Roth, W. T. (1988a). Selective information processing, interoception, and panic attacks. In I. Hand, \& H. U. Wittchen (Eds.), Panic and phobias (Vol. 2, pp. 129-148). Berlin: Springer.

Ehlers, A., Margraf, J., Roth, W. T., Taylor, C. B., \& Birbaumer, N. (1988b). Anxiety induced by false heart rate feedback in patients with panic disorder. Behaviour Research and Therapy, 26, 2-11.

Margraf, J., Taylor, C. B., Ehlers, A., Roth, W. T., \& Agras, W. S. (1987). Panic attacks in the natural environment. Journal of Nervous and Mental Disease, 175, 558-565.

McNally, R. J. (1994). Panic disorder: a critical analysis. New York: Guilford Press.

Pennebaker, J. W. (1982). The physiology of physical symptoms. New York: Springer.

Peterson, R. A., \& Reiss, S. (1992). Anxiety Sensitivity Index Manual (2nd ed.). Worthington, OH: International Diagnostic Systems. Schandry, R. (1981). Heartbeat perception and emotional experience. Psychophysiology, 18, 483-488.

Schandry, R., Bestler, M., \& Montoya, P. (1993). On the relationship between cardiodynamics and heartbeat perception. Psychophysiology, 30, 467-475.

Spielberger, C. D., Gorusch, A., \& Lushen, R. (1970). The State-Trait Anxiety Inventory. Palo Alto, CA: Consulting Psychologist Press.

Tabachnick, B. G., \& Fidell, L. S. (1989). Using multivariate statistics (2nd ed.) New York: Harper-Collins.

Van der Does, A. J. W., Van Dyck, R., \& Spinhoven, Ph. (1997). Accurate heartbeat perception in panic disorder: fact ànd artefact. Journal of Affective Disorders, 43, 121-130.

Van der Does, A. J. W., Van Dyck, R., \& Spinhoven, Ph. (1998). No heart feelings: reply to Ehlers. Journal of Affective Disorders, 49 , $245-247$. 